\title{
Potencial de biodegradación de DDT y sus metabolitos en suelos agrícolas de Chinandega
}

\section{Carlos E. Noguera-Solís y Jorge A. Huete-Pérez}

Centro de Biología Molecular (CBM). Facultad de Ciencia, Tecnología y Ambiente. Universidad Centroamericana, Rotonda Rubén Darío, 500 mts al oeste, Apto. Postal 69. Managua, Nicaragua. Tel: 278-3923 Ext. 1189.Correo electrónico: ce.noguera@gmail.com, $\underline{\text { huete@ns.uca. }}$ $\underline{\text { edu.ni }}$

Recibido: septiembre de 2008 / Aceptado: octubre de 2008

LA PRESENTE INVESTIGACIÓN EVALUÓ EL POTENCIAL DE BIODEGRADACIÓN DEL 1,1,1-Tricloro2,2-bis(4-clorofenil)etano (DDT) y sus metabolitos en suelos agrícolas pertenecientes a fincas del municipio de El Viejo, Chinandega, a través de dos ensayos: ensayo de biodegradación ex situ y ensayo de búsqueda e identificación de microorganismos capaces de biodegradar DDT. En el primer ensayo se determinó el porcentaje de biodegradación, bajo condiciones idóneas, durante un período de 100 días en diferentes lotes de suelo con diferentes aditivos (cachaza y estiércol) a través de la aplicación de cepas microbianas comerciales Environoc 201®. De ello se obtuvo como resultado un porcentaje de biodegradación mayor para el lote que no presentaba aditivos orgánicos, siendo éste de 23,92\%. En el ensayo de búsqueda e identificación de bacterias se lograron aislar e identificar cepas microbianas nativas de los suelos agrícolas objeto de estudio utilizando medios de cultivo con DDT como única fuente de carbono y energía. Como resultado se lograron identificar los siguientes microorganismos: Pseudomonas sp., Streptomyces sp., Phanerochaete chrysosporium y levaduras.

Palabras clave: biodegradación ex situ / DDT / Environoc 201® / NicaraguaChinandega

\section{Introducción}

El 1,1,1-Tricloro-2,2-bis(4-clorofenil)etano (DDT) (Kamanavalli y Ninnekar, 2004) es un insecticida perteneciente a la familia de los organoclorados. Por su alta toxicidad, persistencia y capacidad bioacumulativa, tiene implicaciones desfavorables para el ambiente, la sociedad y la economía. En Nicaragua fue utilizado a gran escala a partir de la explosión del cultivo del algodón entre los años 70 y 80 (EMBUSA, 1999). A pesar de que su utilización ha disminuido en gran medida en los últimos 20 años, aún se encuentran concentraciones significativas de DDT y sus metabolitos en los suelos agrícolas y el agua.

Sus efectos sobre la biota acuática, suelos, agua y cadena alimenticia, así como sus efectos bioacumulativos que provocan afectaciones al sistema nervioso (niveles altos de la enzima 
colinesterasa) y que pueden provocar incluso la muerte (ATSDR, 2002), han motivado la realización de investigaciones con el fin de reducir su impacto.

La presente investigación pretende evaluar el potencial de biodegradación de DDT en suelos agrícolas ubicados en la "Zona Sur Los Millonarios" en el municipio de El Viejo, departamento de Chinandega, donde se han encontrado concentraciones muy elevadas de DDT (Pantaleón, 2007). La biodegradación no es más que la biotransformación de la estructura de compuestos orgánicos mediante la ruptura de los enlaces moleculares (Álvarez y Guevara, 2003).

Para llevar a cabo la evaluación del potencial de biodegradación se efectuó un ensayo de biodegradación en suelos pertenecientes a la finca "El Bosque" utilizando cepas microbianas comerciales del tipo Environoc 201® (E201®) durante un período de 100 días. A lo largo de este período se monitorearon variables ambientales ( $\mathrm{ph}$, humedad y $\mathrm{T}_{\mathrm{w}}$ ) que sirvieron para controlar el crecimiento microbiano asociado con la actividad biodegradadora sobre el DDT. La segunda parte de la investigación (segundo ensayo) buscó cultivar, aislar e identificar bacterias nativas de los suelos del resto de las fincas, capaces de tolerar y biodegradar DDT.

El estudio pretende servir como marco de referencia para el desarrollo de futuras investigaciones que pretendan identificar microorganismos capaces de biodegradar el DDT u otro tipo de plaguicida, a través del uso de una variedad de medios selectivos de cultivo. Por su parte, un ensayo ex situ constituye un buen punto de partida para la proposición de futuros proyectos de biorremediación in situ a gran escala que disminuirían el grado de contaminación por DDT y las correspondientes afectaciones a la salud de la población.

\section{Objetivos}

El objetivo general planteado fue evaluar el potencial de biodegradación de DDT en suelos agrícolas pertenecientes a la "Zona Sur Los Millonarios” del Ingenio Monte Rosa. También nos planteamos los siguientes objetivos específicos:

- Establecer las condiciones idóneas para remediar los suelos de la "Zona Sur Los Millonarios” del Ingenio Monte Rosa por bioaumentación ex situ.

- Determinar el porcentaje de biodegradación de DDT en un intervalo de tiempo definido, mediante la aplicación de cepas microbianas y residuos orgánicos.

- Aislar e identificar microorganismos capaces de tolerar y biodegradar DDT bajo determinadas condiciones.

- Determinar las concentraciones en las que se de mayor crecimiento de microorganismos capaces de tolerar y/o biodegradar DDT. 


\section{Metodología}

Para evaluar el potencial de biodegradación de la "Zona Sur Los Millonarios" dividimos el estudio en dos ensayos: ensayo de biodegradación y ensayo de búsqueda e identificación de microorganismos.

En el ensayo de biodegradación se establecieron las condiciones idóneas para remediar los suelos del Ingenio Monte Rosa al utilizar microorganismos comerciales, Environoc 201 (E201®), por un período de 100 días. Con esto se evaluó la posibilidad de implementar un proyecto de biorremediación dentro del Ingenio Monte Rosa.

Por otra parte, se aislaron e identificaron microorganismos capaces de tolerar y biodegradar DDT en medios con DDT como fuente de carbono. El DDT se aplicó con diversas concentraciones para determinar en qué concentración se observaba mayor crecimiento de colonias.

\section{1 Área de estudio}

Las fincas objeto de estudio se ubicaron en un área de 7, 175.26 ha. (Ilustración 1) conocida como la "Zona Sur Los Millonarios" en el departamento de Chinandega, municipio de El Viejo (Pantaleón, 2007). Las fincas seleccionadas fueron rociadas por DDT durante el cultivo del algodón, antes de 1990. Se seleccionó la Zona Sur por considerarse la zona más contaminada por DDT y sus metabolitos. Esto según un estudio previo analizado por cromatografía de gases por detección de captura de electrones (GC-ECD) (Pantaleón, 2007), realizado en la mayoría de las fincas de esta zona.

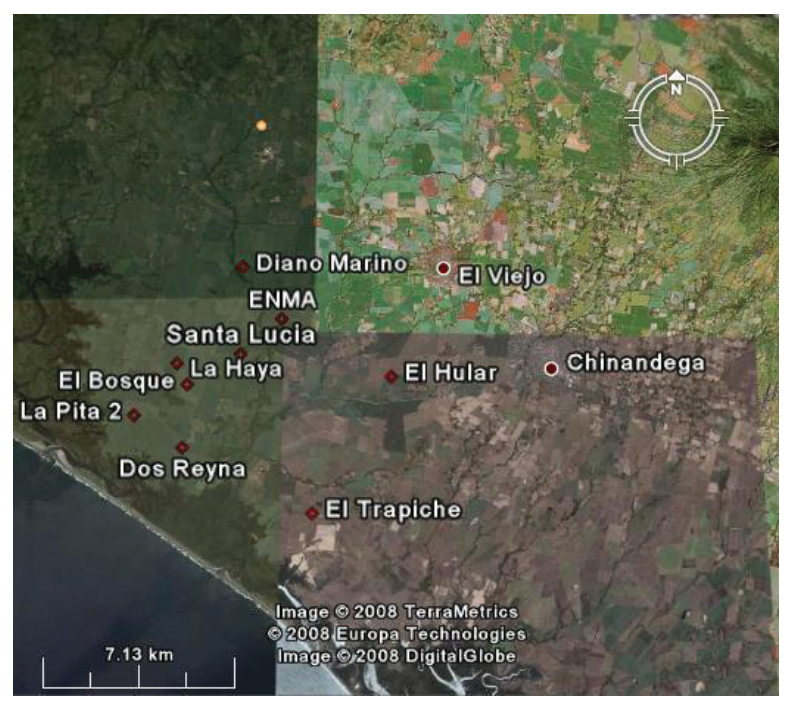

Ilustración 1. Ubicación de las fincas en la zona de estudio (El Viejo, Chinandega) Fuente: Terra Metrics (2008), Europa Technologies (2008), Digital Globe (2008). 


\subsection{Muestreo}

La población corresponde a los suelos de los estratos dentro de las fincas seleccionadas y está definida por los transectos de la ruta de recorrido de muestreo (Mejías y Jerez, 2006).

\subsubsection{Criterio de selección de puntos de muestreo}

Se seleccionaron los puntos de muestreo por finca, es decir, cada finca representa una muestra. Se seleccionaron las fincas que en algún momento fueron rociadas por DDT, escogiendo aquellas donde previamente se hubieran realizado análisis de presencia y concentración de DDT por cromatografía de gases por detección de captura de electrones (GC-ECD). Se seleccionaron fincas con diversas concentraciones de DDT. Cada muestra de cada finca contiene una serie de sub-muestras tomadas cada 20 ha. El Cuadro 1 describe la concentración de DDT y el área de cada finca seleccionada.

Cuadro 1. Fincas muestreadas perteneciente a la “Zona Sur Los Millonarios”.

\begin{tabular}{|c|c|c|}
\hline Finca & $\begin{array}{c}\text { Concentración de } \\
\text { DDT (ppb) }\end{array}$ & $\begin{array}{c}\text { Área de la } \\
\text { finca (ha.) }\end{array}$ \\
\hline El Bosque & 906.90 & 131.39 \\
\hline El Trapiche & 487.20 & 6.98 \\
\hline Santa Lucía y La Haya & 410.40 & 194.99 \\
\hline El Hular & 143.50 & 161.51 \\
\hline ENMA & 116.00 & 55.90 \\
\hline Diano Marino & 98.30 & 125.22 \\
\hline Dos Reyna & 90.50 & 174.93 \\
\hline La Pita 2 & 60.00 & 122.82 \\
\hline \multicolumn{2}{|c|}{ Total } & 973.74 \\
\hline
\end{tabular}

Se realizó un segundo muestreo de la finca "El Bosque" utilizando los mismos puntos georeferenciados, con la diferencia de que las muestras aumentaron en volumen para un total de $3.00 \mathrm{~m}^{3}$ ó $0.43 \mathrm{~m}^{3}$ por cada una de las siete sub-muestras.

\subsubsection{Recolección, preservación e identificación de muestras}

A continuación se describe la metodología seguida para la recolección, preservación e identificación de muestras para el ensayo de biodegradación y el ensayo de búsqueda e identificación de microorganismos. 


\section{Ensayo de biodegradación}

El muestreo de suelos realizado en la finca "El Bosque" corresponde al ensayo de biodegradación en el cual se trazaron transectos a lo interno de cada finca (Mejías y Jerez, 2006). A lo largo de éstos se fijaron puntos de muestreo donde se cavaron calicatas de $1.00 \mathrm{~m} \times 1.00 \mathrm{~m}$ x $0.30 \mathrm{~m}$. Se llenaron ocho sacos de suelo por cada una, siendo abiertas a una calicata por cada 20.00 ha. En total se muestrearon siete puntos (georeferenciados) obteniéndose $3.00 \mathrm{~m}^{3}$ de suelo como una muestra compuesta.

\section{Ensayo de búsqueda e identificación de microorganismos}

Las sub-muestras se recolectaron por medio de calicatas con dimensiones de $0.10 \mathrm{~m} \times 0.10 \mathrm{~m}$ x $0.30 \mathrm{~m}$. Cada muestra fue recolectada en bolsas plásticas (zip-lock) libres de contaminantes o sustancias que pudieran alterar el $\mathrm{pH}$ de la muestra. Se anotó el $\mathrm{pH}$ y temperatura de la muestra in situ. Se anotaron las coordenadas de cada sub-muestra en una hoja de campo. No fue necesario ajustar el $\mathrm{pH}$ de las muestras, ya que se encontraron entre un $\mathrm{pH}$ de $5.0-$ 9.0 (EPA, 1984).

\subsection{Ensayo de biodegradación}

Con el fin de determinar qué aditivos (microorganismos, abonos, estiércol, bagazo, etc.) podrían aumentar la velocidad de degradación del DDT, y por ende el porcentaje de degradación, se dividió una muestra compuesta de $3 \mathrm{~m}^{3}$ de suelo en seis partes, cada una con un volumen de $0.5 \mathrm{~m}^{3}$. Para realizar este ensayo se utilizó la cepa microbiana Environoc $201 \circledR$, Environbrew®, materia orgánica (cachaza y estiércol bovino), agua residual y agua deionizada y destilada.

\subsubsection{Análisis}

A cada uno de los lotes se les dio seguimiento por 100 días (del día 0 al 99) con el fin de determinar los factores que influyeron en la degradación de DDT. Para ello se determinó la concentración inicial de DDT, recuento de poblaciones microbianas, textura del suelo, humedad (\%), temperatura, $\mathrm{pH}$ y concentración de micro y macroelementos.

\subsubsection{Degradación de DDT}

Se determinó la concentración de DDT en el tiempo por medio de GC-ECD. Se utilizó un cromatógrafo Varian modelo 3400 con el detector a una temperatura de $350^{\circ} \mathrm{C}$ y el inyector a $220^{\circ} \mathrm{C}$. Este análisis se realizó en el Laboratorio Nacional de Residuos Orgánicos, del Ministerio Agropecuario y Forestal (MAGFOR). Los análisis se realizaron los días 70 y 99 únicamente para los lotes 2,3 y 4 . Se realizó una muestra inicial (día 0) y un análisis el día 99 para los controles 1 y 2 con el fin de determinar el posible efecto de dilución que pudo haber ocurrido. Debido al alto costo de cada análisis, se realizó una muestra compuesta. En el Cuadro 2 se describe la composición de cada uno de los lotes y controles. 
Cuadro 2. Composición de cada uno de los lotes y controles utilizados en el ensayo de biodegradación

\begin{tabular}{|c|c|}
\hline Lote / Control & Descripción \\
\hline Lote 1 & $\begin{array}{l}\text { Se tomaron } 0.50 \mathrm{~m}^{3} \text { de suelo contaminado con DDT. No se adicionó } \\
\text { ningún aditivo. }\end{array}$ \\
\hline Lote 2 & $\begin{array}{l}\text { Se tomaron } 0.50 \mathrm{~m}^{3} \text { de suelo contaminado con DDT. Se le aplicó En- } \\
\text { vironoc } 201 \AA \text {. }\end{array}$ \\
\hline Lote 3 & $\begin{array}{l}\text { Se tomaron } 0.50 \mathrm{~m}^{3} \text { de suelo contaminado con DDT y } 0.20 \mathrm{~m}^{3} \text { de es- } \\
\text { tiércol bovino. Se le aplicó Environoc } 201 \circledR \text {. }\end{array}$ \\
\hline Lote 4 & $\begin{array}{l}\text { Se tomaron } 0.50 \mathrm{~m}^{3} \text { de suelo contaminado con DDT y } 0.20 \mathrm{~m}^{3} \text { de es- } \\
\text { tiércol bovino. }\end{array}$ \\
\hline Control 1 & $\begin{array}{l}\text { Se tomaron } 0.50 \mathrm{~m}^{3} \text { de suelo contaminado con DDT, } 0.10 \mathrm{~m}^{3} \text { de ca- } \\
\text { chaza y } 5 \text { galones de agua residual. }\end{array}$ \\
\hline Control 2 & $\begin{array}{l}\text { Se tomaron } 0.50 \mathrm{~m}^{3} \text { de suelo contaminado con DDT, } 0.10 \mathrm{~m}^{3} \text { de ca- } \\
\text { chaza y } 5 \text { galones de agua residual. Se aplicó Environoc } 201 \AA \text {. }\end{array}$ \\
\hline
\end{tabular}

\subsubsection{Indicadores}

Los controles son indicadores que fueron útiles para determinar el posible estado de degradación de DDT sin necesidad de realizar los análisis de GC-ECD para organoclorados. En el Cuadro 3 se describen los indicadores utilizados en este ensayo.

Cuadro 3. Indicadores utilizados para el ensayo de biodegradación.

\begin{tabular}{|l|l|}
\hline Indicador & Descripción \\
\hline $\begin{array}{l}\text { Unidades formadoras de } \\
\text { colonias (UFC) }\end{array}$ & Se midió el día $0,10,50$ y 99. \\
\hline $\begin{array}{l}\text { Temperatura ambiente } \\
\left(\mathrm{T}_{\mathrm{amb}}\right)\end{array}$ & Se midió al menos dos veces por semana. \\
\hline $\begin{array}{l}\text { Temperatura del suelo } \\
\left(\mathrm{T}_{\text {suelo }}\right)\end{array}$ & $\begin{array}{l}\text { Se midió al menos dos veces por semana. Corresponde a la } \\
\text { temperatura interna del suelo. }\end{array}$ \\
\hline $\begin{array}{l}\text { Temperatura de trabajo } \\
\left(\mathrm{T}_{\mathrm{w}}\right)\end{array}$ & Se midió al menos dos veces por semana. \\
\hline Humedad $(\%)$ & Se midió cada vez que se aplicó el inóculo microbiano. \\
\hline pH & Se midió cada vez que se aplicó el inóculo microbiano. \\
\hline
\end{tabular}




\subsubsection{Requerimientos mínimos}

Para que los microorganismos (Environoc 201®) contaran con un medio benéfico, necesitaron de los siguientes requerimientos mínimos.

Oxígeno: Se mezcló el suelo al menos una vez por semana con el fin de asegurar la oxigenación mínima del suelo (Biodyne, s.f.).

Humedad: La humedad del suelo tuvo que mantenerse alrededor de un $22 \%$ y siempre menor a un $40 \%$. El suelo no tuvo que estar seco (polvo) ni tan húmedo que conllevara lixiviados (Biodyne, s.f.).

Macroelementos (nutrientes): Se proporcionó al suelo una dosis mínima de nutrientes relación carbono $(\mathrm{C})$ y nitrógeno $(\mathrm{N})$ - con el fin de facilitar el crecimiento microbiano. En caso de que la relación C:N fuera mayor a 100:2 ó 50:1, no sería necesario aplicar fertilizante (Biodyne, s.f.).

\subsubsection{Identificación de microorganismos}

Se identificaron microorganismos con el fin de observar la incidencia de los mismos en la degradación de DDT, de tal forma que se facilitara la identificación de las especies que aumentaron o disminuyeron a lo largo del tratamiento. Se tomaron $10 \mathrm{~g}$ de la muestra (suelo), se mezclaron en un Erlenmeyer con $99 \mathrm{~mL}$ de solución salina ( $\mathrm{NaCl})$, agitándose por varios minutos. Se preparó una serie de diluciones a partir de la primera dilución $10^{-2}$ hasta obtener la dilución $10^{-6}$. Luego se sembró por triplicado en placas de Petri con medio nutritivo a partir de la dilución $10^{-5}$. Las placas fueron incubadas de forma invertida a $30^{\circ} \mathrm{C}$. A partir de 24 horas se procedió al recuento de colonias, reportándose como unidades formadoras de colonias (UFC)/por gramo de suelo. En la presente investigación se entiende UFC como UFC/g. Este procedimiento fue realizado por el Laboratorio de Microbiología de la Universidad Nacional Agraria (UNA), utilizando protocolos propios de la UNA.

\subsubsection{Resumen de la metodología}

En la Ilustración 2 se puede observar un resumen de la metodología utilizada en el ensayo de biodegradación. Se observan los indicadores utilizados, el seguimiento y la composición de los lotes. 


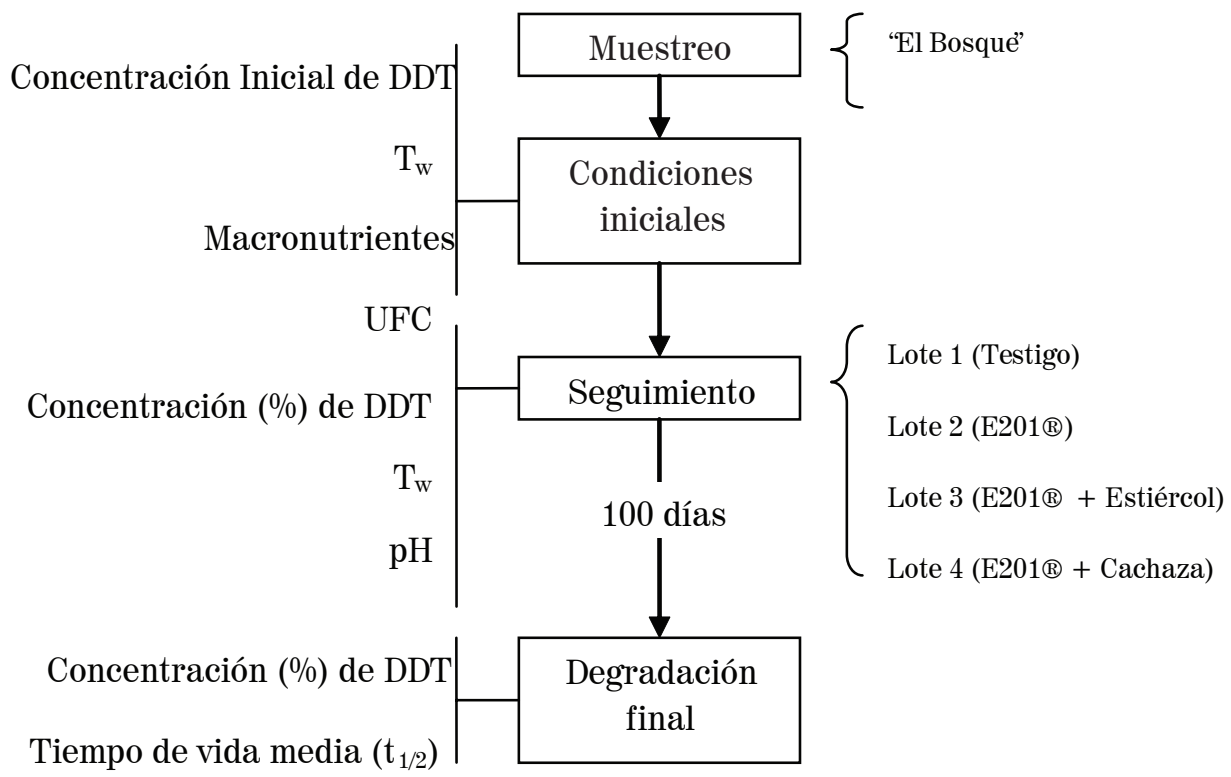

Ilustración 2. Resumen de la metodología utilizada para el ensayo de biodegradación

\subsection{Ensayo de búsqueda y aislamiento de microorganismos}

Con el fin de determinar la concentración de DDT donde existe mayor crecimiento microbiano -y al mismo tiempo asilar microorganismos capaces de tolerar y/o degradar DDT- se aislaron colonias puras como UFC o como unidades de colonias degradadoras (UCD) provenientes de las fincas muestreadas. Se utilizaron los siguientes reactivos: DDT puro (Supelco) facilitado por el Ministerio de Salud (MINSA), $\mathrm{K}_{2} \mathrm{HPO}_{4}, \mathrm{KH}_{2} \mathrm{PO}_{4}, \mathrm{NH}_{4} \mathrm{SO}_{4}$, $\mathrm{MgSO}_{4} \cdot 7 \mathrm{H}_{2} \mathrm{O}, \mathrm{CaCl}_{2} \cdot 2 \mathrm{H}_{2} \mathrm{O}, \mathrm{MnSO}_{4} \cdot \mathrm{H}_{2} \mathrm{O}, \mathrm{FeSO}_{4}, \mathrm{NaMoO}_{4} \cdot 2 \mathrm{H}_{2} \mathrm{O}, \mathrm{NaCl}$ y Ägar Base.

\subsubsection{Aislamiento}

Se colocaron 5.00g de tierra de cada muestra en un tubo de ensayo para luego agregar 50.00 $\mathrm{mL}$ de solución $\mathrm{NaCl}$ al 1\%. Se homogenizó la muestra y se midió pH (Carrillo et al., 2004).

Se utilizaron medios no nutritivos para el crecimiento de microorganismos, teniendo como única fuente de carbono el DDT puro (Carrillo et al., 2004) y sales minerales con la siguiente composición en $\mathrm{g} / \mathrm{L}: \mathrm{K}_{2} \mathrm{HPO}_{4}, 1.73 ; \mathrm{KH}_{2} \mathrm{PO}_{4}, 0.68 ; \mathrm{NH}_{4} \mathrm{SO}_{4}, 1.0 ; \mathrm{MgSO}_{4} 7 \mathrm{H}_{2} 0,0.1$; $\mathrm{CaCl} 2 \mathrm{H}_{2} \mathrm{O}, 0.02 ; \mathrm{MnSO}_{4} \cdot \mathrm{H}_{2} \mathrm{O}, 0.03 ; \mathrm{FeSO}_{4}, 0.03$ y $\mathrm{NaMoO}_{4} \cdot 2 \mathrm{H}_{2} \mathrm{O}, 0.06$. Éstos se esterilizaron a $121^{\circ} \mathrm{C}$ por 15 minutos (Stanlake y Finn, 1982). Se planteó la posibilidad de que, en caso que el compuesto xenobiótico no sustentara el crecimiento de microorganismos, se utilizarían medios enriquecidos con un análogo biodegradable como fenol o bifenilo (Focht y Brunner, 1985) con el fin de facilitar su crecimiento (Atlas y Bartha, 2002). 


\subsubsection{Unidades formadoras de colonias (UFC)}

Se tomó una alícuota de 1/10 de cada una de las muestras de suelo en una solución de $\mathrm{NaCl}$ al 100\%, siguiendo una serie de diluciones entre 10-5 hasta 10-8. Luego fueron plateadas en platos con agar nutritivo Luria Agar Base (LB) sólido. Todos los platos fueron inoculados a una temperatura de $37.00^{\circ} \mathrm{C}$ por $48-96$ horas. Las colonias fueron leídas como unidades formadoras de colonias (UFC), entendiéndose UFC como UFC/g. Como referencia, se analizaron las UFC en el Laboratorio de Microbiología de la Universidad Nacional Agraria (UNA).

\subsubsection{Estudio de tolerancia o unidades de colonias degradadoras (UCD)}

Con el fin de evaluar la tolerancia de DDT para las diferentes poblaciones de microorganismos, se realizó un conteo de número de colonias degradadoras. Se tomó una alícuota en 1/10 mL de $\mathrm{NaCl}$ al $1.00 \%$, siguiendo una serie de diluciones entre $10^{-3}$ hasta $10^{-5}$ que luego fueron plateadas en platos con agar no nutritivo (NNA) sólido (Biodyne, s.f.). Los platos fueron rociados por cuatro concentraciones diferentes, $100,300,600$ y 1000 ppb. Se incubaron a una temperatura de $37^{\circ} \mathrm{C}$ por 48 horas. Como resultado se obtuvieron cultivos mixtos a los que se les realizó una media aritmética entre las tres réplicas midiéndose como UCD, entendiéndose UCD como UCD/g de suelo (Biodyne, s.f.). Se utilizó un blanco por cada serie de diluciones. Los microorganismos de interés se aislaron posteriormente en la identificación.

\subsubsection{Identificación de microorganismos}

A partir de los diferentes cultivos mixtos se aislaron cepas puras. Éstas se platearon en placas con agar enriquecido siguiendo el mismo proceso que el planteado en el inciso 3.4.3. Se realizaron cinco análisis por cada concentración de DDT (100, 300, 600 y 1000 ppb) para un total de veinte análisis por finca. Las cepas que lograron desarrollarse adecuadamente pasaron al proceso de identificación en el que se utilizaron los criterios morfológicos (forma, tinción de Gram, movilidad, pigmentación) y bioquímicos (prueba de oxidación-fermentación, medio O/F glucosa, presencia de enzimas catalasa y/o oxidasa) (Madigan et al., 2004). El proceso de identificación se realizó en el Laboratorio de Microbiología de la Universidad Nacional Agraria (UNA), siguiendo protocolos propios de la UNA.

\subsubsection{Resumen de la metodología}

En la Ilustración 3 se observa un resumen de la metodología utilizada en el ensayo de búsqueda y aislamiento de microorganismos. Se observan los indicadores utilizados, métodos y análisis utilizados. 


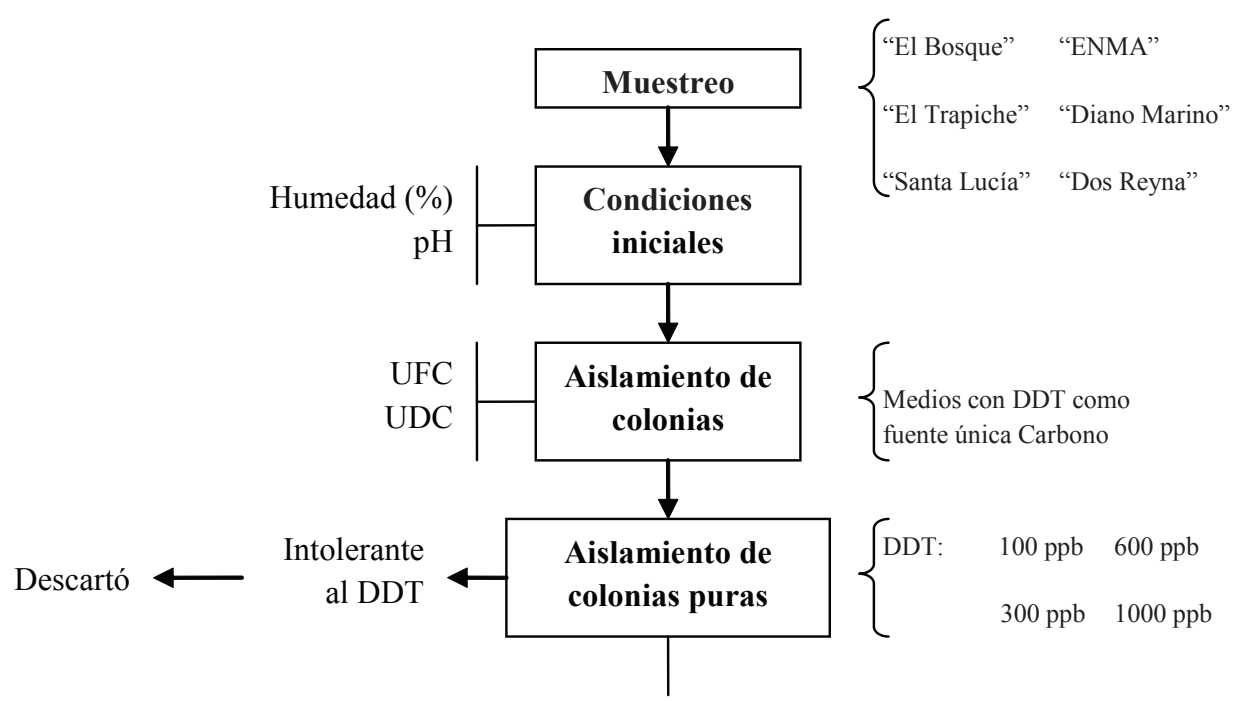

Tolerante al DDT

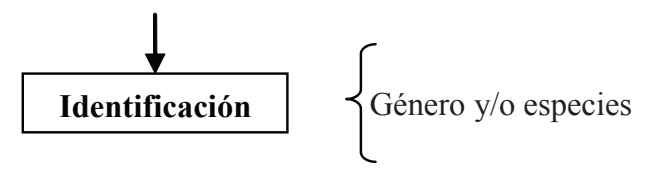

Ilustración 3. Resumen de la metodología utilizada para el ensayo de búsqueda y asilamiento de microorganismos

\subsection{Análisis y procesamiento de la información}

Para facilitar el análisis de los resultados se utilizaron fichas a lo largo del muestreo y recolección de datos. Además se utilizó la bibliografía existente para comparar la veracidad de nuestros resultados. Los resultados fueron analizados estadísticamente por medio del programa Excel versión 2003. Los cálculos estadísticos variaron desde regresión lineal, factor de correlación de Pearson (r), promedio o media aritmética, desviación estándar, error estadístico, pendiente (m), intersección (x) y sumatoria.

Con fines aclaratorios, se utilizó regresión lineal teniendo a $\mathrm{Y}$ como variable en dependencia de una variable independiente X. Ésta se utilizó en las proyecciones de degradación (\%) de DDT en el tiempo (x). Por otro lado, se utilizó el cálculo de correlación de Pearson (r) o coeficiente producto momento de Pearson cuando el fin era encontrar si dos variables son independientes o covarían entre sí (no se expresaron como variable independiente o dependiente) (Pérez, 2004).

El coeficiente de Pearson (r) se dividió en muy bajo (0.00 - 0.20), bajo (0.21 - 0.40), moderado $(0.41-0.60)$, alto $(0.61-0.80)$ y muy alto $(0.81-1.00)$ (Gagel et al., 2007; Hill et al., 1995).

Para los cálculos de error se utilizaron dos términos: desviación estándar y error estadístico. El error estadístico se utilizó en el cálculo de medias de muestras del mismo tamaño de una 
población, tomando en cuenta los valores originales (Tw, pH y humedad (\%)), mientras que el error estándar se utilizó en cálculos que implicaban el error a valores calculados (valores corregidos de la degradación (\%) de DDT y cálculos de correlación) (Skoog et al., 2001).

\section{Resultados y discusión}

\subsection{Ensayo de biodegradación}

A continuación se discuten los resultados obtenidos en el ensayo de biodegradación en la finca "El Bosque".

\subsubsection{Macronutrientes}

La finca "El Bosque" presenta una relación de 20:1 ó 100:5 de C:N, superior a 100:2, por lo cual no fue necesario aplicar nitrógeno. La textura del suelo es franca, predominando partículas de arena (44.80\%), seguida de limo (30.00\%) y por último arcilla $(25.20 \%)$.

\subsubsection{Aplicaciones microbianas: Environoc 201®}

Los cuatro lotes objeto de estudio y los dos controles fueron sometidos a las mismas condiciones para asegurar igualdad de variables ambientales. Se aplicaron $2,661.00 \mathrm{ml}$ de E201® en 13 aplicaciones el mismo día que se oxigenaron los lotes, se aplicó agua y se midieron las condiciones ambientales constantes ( $\mathrm{pH}$ y humedad).

El Lote 1 funge como Testigo al presentar las mismas condiciones ambientales -mas sin la presencia de microorganismos- para determinar el porcentaje de biodegradación atribuido a factores varios de naturaleza no biótica. Si bien al Testigo (Lote 1) no se le aplicaron microorganismos, se homogeneizó y aplicó agua en un volumen que igualara al de la dosis microbiana aplicada en el resto de los lotes. De igual forma, en los Controles 1 y 2 se aplicó agua en una cantidad equivalente a la del Testigo homogeneizándose 13 veces.

Los cálculos de aplicación de E201® se basan en los volúmenes de suelo contaminado por plaguicidas (DDT). De conformidad con los protocolos de aplicación de dosis bacterianas de Biodyne ${ }^{\circledR}$ (Biodyne, 2008), los aditivos no se toman en consideración para la preparación de la dosis.

\subsubsection{Tw, pH, humedad (\%) y UFC}

Se sospechaba que a mayor $\mathrm{T}_{\mathrm{w},}$ la tendencia de biodegradación aumentaría. Este fenómeno no se vio reflejado en el Lote 3, el cual no presentó el mayor porcentaje de biodegradación a lo largo del período de estudio, pero sí las $\mathrm{T}_{\mathrm{w}}$ más altas.

Esto se debió a que la población microbiana del suelo aumentó como resultado de la presencia de estiércol, cuya mineralización conllevó a una liberación elevada de calor. Aunque la T $\mathrm{w}$ es elevada, ello no implica que se deba a efectos de biodegradación de DDT, sino que realmente se debe a efectos de mineralización del estiércol. 
La $\mathrm{T}_{\mathrm{w}}$ para los lotes con cachaza no fue alta pues el aditivo en sí mismo representa una matriz menos nutritiva para los microorganismos por estar compuesta principalmente de celulosa y lignina, que no son tan rápidamente biodegradables como el estiércol.

Si se compara el Lote 1 con el Lote 2 se podrá ver que, a falta de aditivo, el Lote 2 presentó una mayor tendencia a tener una mayor $\mathrm{T}_{\mathrm{w}}$ como resultado de la adición de dosis microbiana. Este incremento se debió únicamente a la actividad microbiana biodegradadora del DDT y a la microfauna nativa del suelo (Pseudomonas sp. y Bacillus sp.).

Aunque la desviación estándar de $\mathrm{T}_{\mathrm{w}}$ en los cuatro lotes fue alta, esto no es un factor que reduzca la capacidad degradadora de los microorganismos, siempre y cuando se mantengan las condiciones de humedad y pH constantes (Beulke et al., 2005).

Los valores de $\mathrm{pH}$ de los lotes se encuentran dentro del rango óptimo de desarrollo de microorganismos. Valores bajos de desviación estándar de los promedios globales indican que el pH del suelo permanece relativamente constante, variando según la heterogeneidad del suelo y la dinámica de sus especies iónicas en la solución.

Los valores de humedad son relativamente constantes, con una desviación estándar baja en relación al promedio. La humedad (\%) nunca estuvo por debajo del $22 \%$ ni por encima de su nivel de saturación (40\%), ya que no se observó lixiviado.

Se observó el crecimiento de Pseudomonas sp, (Kamanavalli y Ninnekar, 2004; Hay y Focht, 1998; Francis et al., 1978; Seubert, 1960), Bacillus sp. (Aislabie et al., 1997; Patil et al., 1970) y Sarcina sp. Las primeras dos especies presentan antecedentes comprobados de biodegradación de DDT. En la muestra inicial se observaron solamente Bacillus sp. A medida que se fueron aplicando las dosis microbianas hubo un aumento de Pseudomonas sp. у Bacillus sp.

Tomando en cuenta todas las especies microbianas, UFC total, el Lote 3 presentó una actividad microbiana aproximadamente dos veces mayor que la del Lote 1, lo cual se debe particularmente a la influencia del estiércol. En el Lote 2, por su parte, se observó que hay una mayor actividad microbiana atribuida a la adición de E201®. Así, se puede ver que en el Lote 3 parte de la actividad microbiana se debe a la microfauna nativa del estiércol y no necesariamente a la adición de E201®. Por ende, la totalidad de estas colonias no logran tolerar ni biodegradar DDT, sólo una parte de ellas.

En el Cuadro 3 se describe un resumen de los promedios de Tw, pH, humedad y UFC en los lotes y controles utilizados para el ensayo de biodegradación. 
Cuadro 3. Resumen de los promedios de las variables

\begin{tabular}{|c|c|c|c|c|}
\hline Lotes & $\mathrm{Tw}\left({ }^{\circ} \mathrm{C}\right)$ & $\mathrm{pH}$ & $\begin{array}{l}\text { Humedad } \\
\quad(\%)\end{array}$ & $\begin{array}{c}\mathrm{UFC} \\
\left(\mathbf{x} 10^{6}\right)\end{array}$ \\
\hline Lote 1 (Testigo) & $0.90 \pm 2.01$ & $6.15 \pm 0.13$ & $26.50 \pm 3.83$ & $14.00 \pm 11.93$ \\
\hline Lote 2 (E201®) & $1.10 \pm 2.30$ & $6.33 \pm 0.28$ & $30.31 \pm 4.30$ & $23.00 \pm 20.05$ \\
\hline $\begin{array}{ll}\text { Lote } & 3 \\
(\mathrm{E} 201 @+\text { Estiércol }) & \end{array}$ & $2.40 \pm 2.30$ & $6.41 \pm 0.32$ & $29.36 \pm 5.77$ & $32.00 \pm 45.57$ \\
\hline $\begin{array}{ll}\text { Lote } & 4 \\
(\mathrm{E} 201 @+\text { Cachaza) }\end{array}$ & $0.70 \pm 2.14$ & $6.38 \pm 0.32$ & $31.10 \pm 3.49$ & $11.00 \pm 1.71$ \\
\hline \multicolumn{5}{|l|}{ Controles } \\
\hline Control 1 (Estiércol) & $1.00 \pm 2.31$ & $6.38 \pm 0.33$ & $31.70 \pm 2.87$ & $12.00 \pm 12.04$ \\
\hline Control 2 (Cachaza) & $-1.00 \pm 2.21$ & $6.32 \pm 0.31$ & $32.05 \pm 2.96$ & $18.00 \pm 14.15$ \\
\hline
\end{tabular}

\subsubsection{Degradación de DDT, $\mathrm{t}_{1 / 2} \mathrm{y}$ tiempo de vida}

A los datos de degradación de DDT (\%) se les aplicó el análisis de regresión lineal debido a que se trataba de un período corto de análisis. El valor del Testigo parece casi inalterable, con un porcentaje de degradación de 2.33\%. Esta pequeña degradación pudo haberse debido a degradación abiótica o bien, a degradación autóctona del suelo inducida por las condiciones de calor a que se vio sometido cada lote al ser cubierto con plástico negro, acompañado de la homogeneización.

El lote que presentó el segundo porcentaje de biodegradación más bajo fue el 4 (E201® + cachaza). Se estima que esta baja degradación se debe a que la cachaza no es un material orgánico con alta presencia de microorganismos capaces de mineralizarla en estas condiciones. A su vez, está compuesta principalmente por celulosa y lignina, cuya degradación, si bien se trata de sustancias biodegradables (Barr y Aust, 1994), es relativamente lenta.

ElLote 3 (E201® + estiércol) fue el segundo en presentar mayor porcentaje de biodegradación. Esto como resultado de la alta presencia de microorganismos que se encuentran en el estiércol. Durante el proceso de mineralización del mismo, el aumento de la temperatura fue mayor, no obstante, lo que determina en verdad el porcentaje de biodegradación es el papel que juegan los microorganismos capacitados para biodegradar DDT.

La razón por la cual el Lote 2, que no contenía aditivos, presentaba el mayor porcentaje de biodegradación con respecto al Lote 3 , puede deberse al hecho de que en este último 
haya acontecido un crecimiento competitivo entre los microorganismos del estiércol y las cepas E201® (Madigan et al., 2004; Atlas y Bartha, 2002) de forma tal que éstas pudieron desarrollarse a su máxima capacidad, biodegradando el DDT a una tasa balanceada.

Se puede advertir que un porcentaje de biodegradación de 25.00\% en 100 días es bastante alto (comparado con el $t_{1 / 2}$ calculado), lo cual sugiere que la degradación no solamente fue aeróbica, sino anaeróbica facultativa, puesto que los lotes estaban apilados y en el fondo de ellos -si bien se homogeneizaban regularmente- el acceso a oxígeno era dificultoso. Se estima que a estas condiciones experimentales ex situ, la biodegradación sufre un comportamiento relativamente lineal, lo que se debe a que el período de tiempo de análisis es corto y las condiciones ambientales permanecen constantes. A medida que el tiempo va aumentando, el comportamiento se vuelve logarítmico.

Según los datos de degradación, se puede predecir el tiempo en el cual se degradará el DDT. Al calcular el $t_{1 / 2}$ se puede determinar realmente cómo influye la aplicación de cepas a lo largo del tiempo, permitiendo decidir si la biodegradación será factible. Así, el Lote 2 se degradaría casi 14 veces más rápidamente que el Testigo. Mientras éste se degradaría a la mitad de su concentración en 209 días, el Testigo haría lo mismo en 8 años.

El tiempo que necesita el DDT y sus metabolitos para biodegradarse hasta 1 ppb se definió como tiempo de vida. En el Cuadro 4 se resumen los promedios obtenidos de la degradación, $\mathrm{t}_{1 / 2} \mathrm{y}$ tiempo de vida. Se puede observar que el Lote 1 (Testigo) se degradaría a 1 ppb en 64.14 años, mientras que el Lote 2 (E201®) haría lo mismo en sólo 4.59 años.

Cuadro 4. Resumen de los promedios de degradación (\%) de DDT, $\mathrm{t}_{1 / 2} \mathrm{y}$ tiempo de vida en los lotes utilizados para el ensayo de biodegradación

\begin{tabular}{|c|c|c|c|c|}
\hline Lotes & $\begin{array}{c}\text { Degradación DDT } \\
(\%)\end{array}$ & $\begin{array}{c}t_{1 / 2} \\
(\operatorname{años})\end{array}$ & t de vida (años) & $\mathbf{X}$ \\
\hline Lote 1 (Testigo) & 2.33 & 7.97 & 64.40 & 1.00 \\
\hline Lote $2(\mathrm{E} 201 \AA)$ & 23.92 & 0.57 & 4.59 & 13.94 \\
\hline $\begin{array}{ll}\text { Lote } & 3 \\
(\mathrm{E} 201 \AA+\text { Estiércol }) & \end{array}$ & 20.19 & 0.86 & 6.89 & 9.26 \\
\hline $\begin{array}{ll}\text { Lote } & 4 \\
(\mathrm{E} 201 \AA+\text { Cachaza }) & \end{array}$ & 12.70 & 1.41 & 11.29 & 5.65 \\
\hline
\end{tabular}

\subsubsection{Factores de correlación}

El Cuadro 5 muestra un resumen de los factores de correlación (r) entre degradación (\%) de DDT, $T_{w}$ y UFC. Se puede observar que la degradación está más sensiblemente relacionada con la actividad microbiana (UFC) $(r=0,45)$ que con la $\mathrm{T}_{\mathrm{w}}(0,20)$ y que, por su parte, $\mathrm{T}_{\mathrm{w}}$ está fuertemente relacionada con UFC. 
Cuadro 5. Factores de Correlación (r) entre degradación (\%) de DDT, Tw y UFC.

\begin{tabular}{|c|c|c|c|}
\cline { 2 - 4 } \multicolumn{1}{c|}{} & Degradación & Tw & UFC \\
\hline Degradación & 1.00 & 0.20 & 0.45 \\
\hline Tw & 0.20 & 1.00 & $\mathbf{0 . 8 6}$ \\
\hline UFC & 0.45 & $\mathbf{0 . 8 6}$ & 1.00 \\
\hline
\end{tabular}

Si bien la relación entre $\mathrm{T}_{\mathrm{w}} \mathrm{y}$ la degradación (\%) es muy baja, $\mathrm{T}_{\mathrm{w}} \mathrm{y}$ la actividad microbiana expresada como UFC sí presenta una correlación muy alta $(\mathrm{r}=0,86)$, lo que indica una sólida tendencia positiva $(\mathrm{m}=0,07)$ donde a mayor $\mathrm{T}_{\mathrm{w}}$ habrá una mayor actividad microbiana (UFC).

La Ilustración 4 muestra la tendencia de la correlación entre $\mathrm{T}_{\mathrm{w}} \mathrm{y}$ UFC, donde al aumentar la temperatura UFC aumenta $\mathrm{T}_{\mathrm{w}}$. Ésto se debe a la estrecha relación que existe entre la actividad (degradadora) microbiana y los cambios de temperatura (Beulke et al., 2005), pudiéndose aseverar que a medida que se eleva la $\mathrm{T}_{\mathrm{w}}$ las reacciones químicas y enzimáticas de la célula son más rápidas, acelerándose el crecimiento o la actividad microbiana (UFC) (Madigan et al., 2004). Según estos resultados, la actividad microbiana (UFC) es el indicador más fiable en la degradación de DDT en suelos.

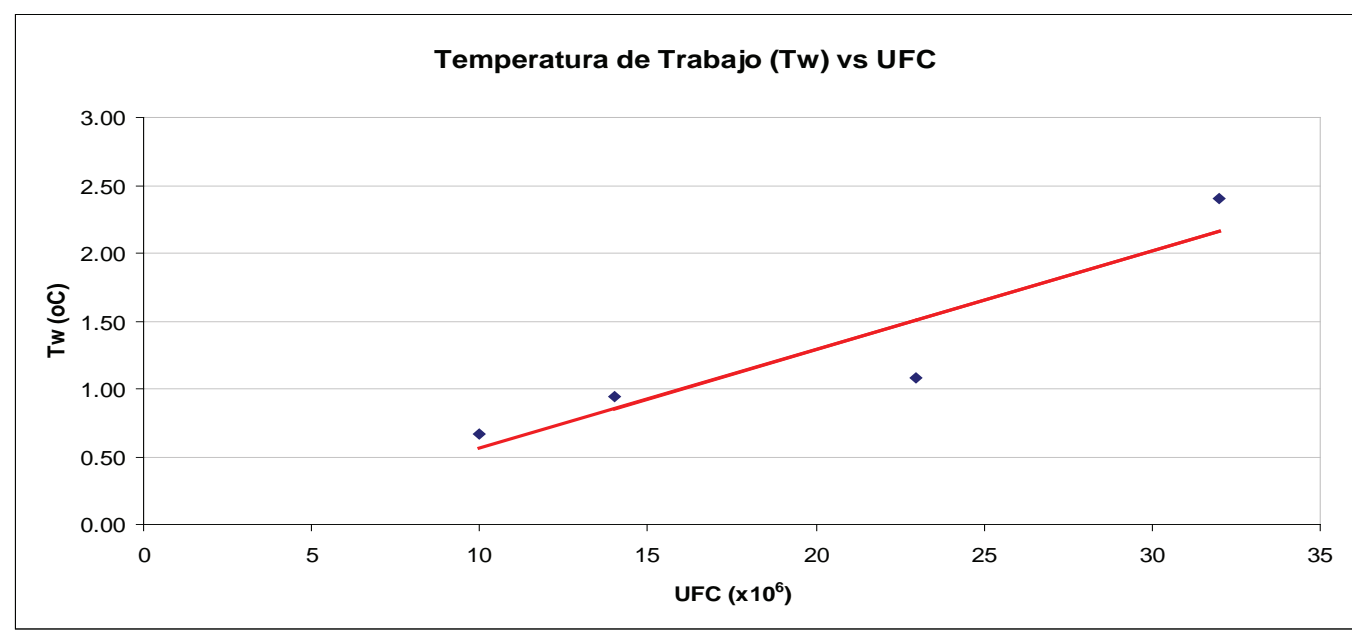

Ilustración 4: Temperatura de trabajo $\left(\mathrm{T}_{\mathrm{w}}\right)$ contra unidades formadoras de colonias (UFC).

\subsubsection{Inversión}

El costo de la inversión se determinó en base a los costos de remediación de $0.5 \mathrm{~m}^{3}$ del Lote 2 (E201). Estos costos no incluyen transporte, maquinaria, impuestos, ni costo por importación del producto. Debido a que el tiempo de vida del DDT puede variar, el costo se calculó en base al tiempo de aplicación del producto (años). 
Los costos para remediar $1.00 \mathrm{~m}^{3}$ de suelo contaminado por DDT son de $\$ 320.00$ anuales y \$26.67 mensuales. Si bien el costo de biorremediar un suelo es más bajo que otras técnicas (Cuadro 6), remediar áreas muy extensas eleva el costo de remediación, especialmente en residuos peligrosos como los pesticidas. Lo más recomendable es utilizar E201® sólo para puntos focales o derrames de plaguicidas en campo, y adicionar nutrientes al suelo, a través del sistema de riego, que ayuden a la microfauna existente a cometabolizar el DDT.

Cuadro 6. Costos de alternativas para tratar suelos contaminados por desechos peligrosos

\begin{tabular}{|c|c|c|}
\hline Tipo de tratamiento & $\begin{array}{c}\text { Costo medio } \\
\qquad\left(\$ / \mathbf{m}^{3}\right)\end{array}$ & $\begin{array}{l}\text { Rango típico del } \\
\text { costo }\left(\$ / \mathbf{m}^{3}\right)\end{array}$ \\
\hline Incineración & 975 & $350-1600$ \\
\hline Relleno sanitario & 350 & $100-600$ \\
\hline Desorción térmica & 125 & $50-200$ \\
\hline Lavado de suelo & 237 & $125-350$ \\
\hline Biorremediación sobre superficie & 95 & $40-150$ \\
\hline
\end{tabular}

Fuente: Álvarez y Guevara (2002).

\subsection{Ensayo de búsqueda y aislamiento de microorganismos}

A continuación se discuten los resultados obtenidos en el ensayo de aislamiento e identificación de microorganismos capaces de biodegradar DDT.

En el Cuadro 7 se observa que las fincas tenían en promedio un $\mathrm{pH}$ de 6.64, teniendo el menor "El Bosque" (6.30) y el mayor "La Haya" (6.90). Los rangos de pH son aceptables, no hubo necesidad de ajustarlos. La humedad en promedio fue de 3.08\%, valores aceptables para el crecimiento microbiano por el método de placas.

Cuadro 7: Condiciones inicial de las fincas muestreadas

\begin{tabular}{|c|c|c|}
\hline Finca & $\mathbf{p H}$ & Humedad (\%) \\
\hline El Bosque & 6.30 & 2.91 \\
\hline El Hular & 6.45 & 3.56 \\
\hline El Trapiche & 6.70 & 3.71 \\
\hline ENMA & 6.67 & 2.78 \\
\hline La Pita 2 & 6.34 & 1.90 \\
\hline Dos Reyna & 6.89 & 3.12 \\
\hline Santa Lucía & 6.61 & 3.00 \\
\hline La Haya & 6.90 & 2.95 \\
\hline Diano Marino & 6.86 & 3.81 \\
\hline Promedio & $\mathbf{6 . 6 4}$ & $\mathbf{3 . 0 8}$ \\
\hline
\end{tabular}




\subsubsection{Unidades de colonias degradadoras (UCD)}

Las fincas en las que se observó una mayor formación de UCD fueron "El Bosque" con 2.71 x 105; "Dos Reyna" con 1.93 x 105; "Santa Lucía" con 1.79 x 105; y "La Pita 2" con 1.75 x $10^{5}$. También se observó que a mayor concentración de DDT aplicado hubo una menor formación de colonias.

A su vez, la proporción de UCD es menor que la de unidades formadoras de colonias (UFC). Esto es lógico, ya que UFC (19.67 x $\left.10^{5}\right)$ expresa la población total de microorganismos, mientras que UCD $\left(1.43 \times 10^{5}\right)$ sólo expresa la población capaz de tolerar o biodegradar DDT. Sin embargo, esto no es aplicable para la finca "Diano Marino" posiblemente porque la población microbiana de esta finca en su mayoría es capaz de tolerar DDT. Con el fin de confirmar la existencia de una población degradadora representativa en la finca "Diano Marino", se deberán realizar análisis más profundos tanto de UCD como de UFC.

\subsubsection{Relación de UCD y concentración de DD'T}

Se observó una fuerte correlación $\left(\mathrm{r}=0,95 ; \mathrm{m}=-72,16 \pm 12,11\right.$; Intersección $(\mathrm{x})=1,79 \mathrm{x} 10^{5}$ $\left.\pm 0,07 \times 10^{5}\right)$ entre la concentración de DDT (\%) aplicada y las unidades de colonias degradadoras (UCD). Esto permite afirmar que a menor concentración de DDT es más fácil para los microorganismos adaptarse a este medio en un corto lapso de tiempo. Esto no quiere decir que no exista la adaptación o bien, que la adaptación sea menor a concentraciones más altas, ya que se ha demostrado que en prolongados períodos de tiempo, las colonias logran adaptarse (Álvarez y Guevara, 2003; Gale, 1952).

La Ilustración 5 muestra la correlación (muy alta) entre UCD y la concentración (\%) de DDT, siendo ésta negativa $(\mathrm{m}=-72,16)$ o inversamente proporcional y una Intersección $(\mathrm{x})$ igual a $1.79 \times 10^{5} \pm 0.07 \times 10^{5}$.

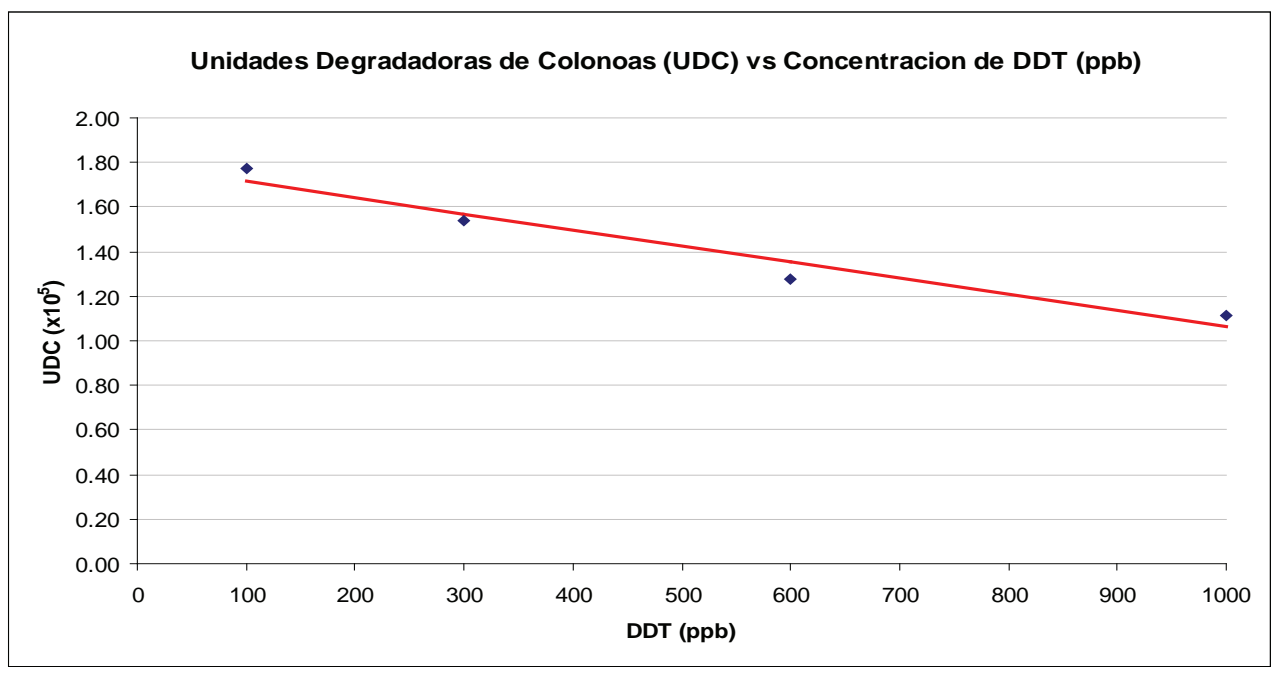

Ilustración 5. Correlación entre UCD y concentración (\%) de DDT 


\subsubsection{Especies aisladas e identificadas}

Se logró identificar una variedad de microorganismos entre los que se encuentran bacterias, hongos, levaduras y actinomicetos. Tanto las bacterias (Pseudomonas sp.) (Kamanavalli y Ninnekar, 2004; Hay y Focht, 1998; Francis et al., 1978; Seubert, 1960), como los actinomicetos (Streptomyces sp.) (Chacke $e t$ al., 1966), los hongos (Aislabie et al., 1997; Bumpus y Aust, 1987) y las levaduras (Kallman y Andrews, 1963) han presentado afinidad por tolerar o degradar plaguicidas, incluyendo el DDT.

Por otro lado, el único microorganismo que presenta afinidad por mineralizar DDT son los hongos, resaltando la especie Phanerochaete chrysosporium (Bumpus y Aust, 1987).

En las Ilustraciones 6 y 7 se puede observar una especie aislada de bacteria (Pseudomonas sp.) y una de hongo (Phanerochaete chrysosporium).

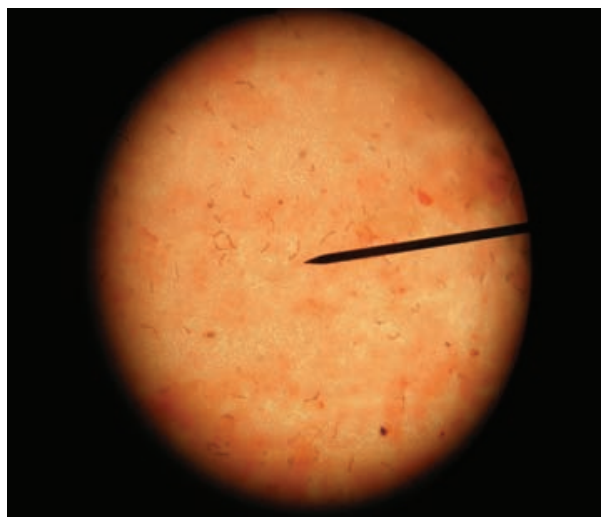

Ilustración 6. Microorganismo identificado: Pseudomonas sp.

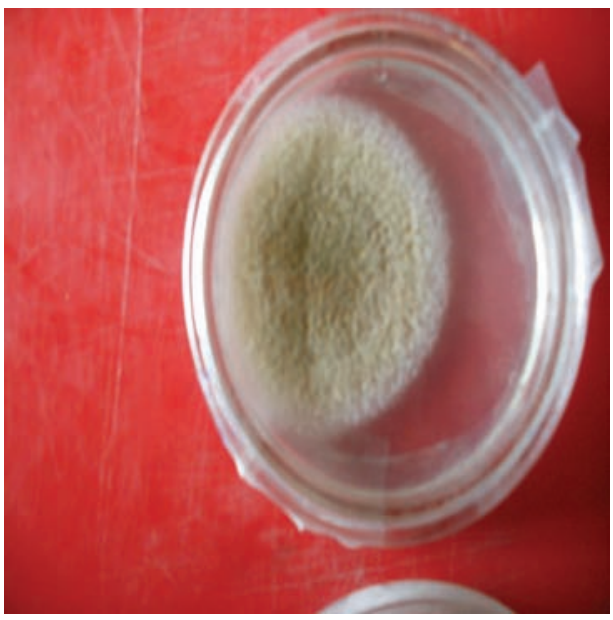

Ilustración 7. Microorganismo identificado: Phanerochaete chrysosporium 


\subsubsection{Relación entre colonias aisladas y concentración de DDT}

En el Cuadro 8 se puede observar que 13 de las 45 colonias que se intentaron aislar a una concentración de 1000 ppb, lograron desarrollarse, tendencia que disminuyó en las concentraciones menores, tanto así que sólo 3 de 45 colonias lograron desarrollarse a una concentración de 1000 ppb. Si bien la relación entre las colonias aisladas y concentración de DDT no formó parte de nuestro estudio, es interesante ver la relación de que a mayor concentración de DDT, hay mayor probabilidad de aislar las colonias $(\mathrm{r}=0.98)$.

Cuadro 8: Colonias aisladas contra concentración de DDT

\begin{tabular}{|c|c|}
\hline Concentración & $\mathrm{N}^{0}$ de colonias aisladas \\
\hline 100 & 3 \\
\hline 300 & 4 \\
\hline 600 & 8 \\
\hline 1000 & 13 \\
\hline Total & 45 \\
\hline
\end{tabular}

La facilidad que tienen los microorganismos para aislarse como cultivos puros en un medio con concentraciones altas de DDT se explica al haber una mayor disponibilidad de éste como fuente de carbono, mientras que a concentraciones menores, existe una menor disponibilidad.

\section{Conclusiones}

El potencial de biodegradación de DDT y sus metabolitos en los suelos fue significativo, ya que se logró biodegradar el DDT en un 24.00\% y se lograron aislar microorganismos capaces de tolerarlo y biodegradarlo.

Las condiciones idóneas para biodegradar los suelos de la finca "El Bosque" se dan con la aplicación de dosis de Environoc $201 @$ inoculadas por 24 horas, con una humedad (22 $40 \%)$ y $\mathrm{pH}(\sim 7)$ óptimos.

El mayor porcentaje de biodegradación de DDT en 100 días correspondió al Lote 2 (Environoc $201 \circledR$ ) con $24.00 \%$, seguido del Lote 3 con $20.00 \%$ (Environoc $201 \circledR$ y estiércol) y del Lote 4 con $13.00 \%$ (Environoc $201 \AA$ y cachaza).

La temperatura de trabajo $\left(\mathrm{T}_{\mathrm{w}}\right)$ no se relaciona fuertemente con el porcentaje de biodegradación de DDT $(\mathrm{r}=0.20)$, pero sí se relaciona sólidamente con la actividad microbiana en el suelo (UFC) $(r=0.86)$.

Se aislaron 28 colonias puras de microorganismos capaces de tolerar o biodegradar DDT, identificándose bacterias (Pseudomonas sp.), actinomicetos (Streptomyces sp.), hongos (Phanerochaete chrysosporium) y levaduras. 
El mayor crecimiento de colonias tolerantes o degradadoras de DDT (UCD) se produjo a una concentración 100 ppb de DDT. Por su parte, se observó una mayor facilidad de aislamiento de colonias puras, capaces de tolerar o degradar DDT, a una concentración de 1000 ppb de DDT.

El costo de biorremediar suelos de uso agrícola contaminados por plaguicidas, en la Zona Sur "Los Millonarios" se estima de $\$ 360 \mathrm{~m}^{3} /$ año y $\$ 26.66 \mathrm{~m}^{3} / \mathrm{mes}$, lo que se encuentra relativamente más económico que otras técnicas.

\section{Recomendaciones}

- Acordar con el Ministerio del Ambiente y de los Recursos Naturales (MARENA) los límites máximos permisibles (lmp) para suelos con fines agrícolas con presencia de plaguicidas. Esto facilitará plasmar las metas ambientales dentro del Sistema de Gestión Ambiental (SGA) y evaluarlas anualmente.

- Utilizar E201® en los meses que sea más factible regar los suelos, es decir, en tiempo fuera de cultivo. Así se puede dar tratamiento a los suelos tres meses al año, evaluando anualmente la degradación hasta llegar a una concentración lo suficientemente baja.

- Utilizar cepas comerciales (E201®) en puntos focales de contaminación, es decir, en derrames accidentales de pesticidas que se den en los almacenes, aeródromos agrícolas o cerca de alguna población y cuerpo de agua.

- Para posibles estudios de biodegradación de DDT ex situ se recomienda realizar un ensayo conjunto bajo condiciones aeróbicas y anaeróbicas intercaladas, o bien, que consideren un ambiente aeróbico o anaeróbico facultativo para analizar el potencial de E201® (o de cualquier otra cepa microbiana comercial) en otro tipo de medio.

- Para evitar la disminución de la actividad biodegradadora de E201®, se recomienda utilizar aditivos que no presenten en su matriz microorganismos asociados a actividades de descomposición de materia orgánica que puedan entrar en competencia con microorganismos biodegradadores.

- Para profundizar en el estudio de biodegradación, se recomienda realizar análisis individuales en los que se someta a cada cepa identificada a una concentración inicial de DDT y se evalúe, consecuentemente, durante un período de tiempo establecido y bajo condiciones ambientales controladas, el porcentaje de DDT biodegradado asociado a la actividad biodegradadora del microorganismo objeto de estudio.

\section{Agradecimientos}

Agradecemos a muchas personas e instituciones que hicieron posible esta investigación. Al Centro de Biología Molecular (CBM-UCA) por fondos provenientes de New England Biolabs, Inc (NEB, EE.UU.) y de Fondos para la Investigación de la Universidad Centroamericana (FIUCA); además por el apoyo técnico brindado. A Adriana Guzmán, Indiana García y Enrique 
Campbell por la revisión y recomendaciones durante la investigación. A los colaboradores del sitio de muestreo: Carlos García, Gustavo Ralda, Dalia Jiménez, Elvin Arróliga, Miguel Oviedo, Marlon Guzmán y Winston Palma. Al MINSA, MAGFOR y UNA, por su colaboración, sobresaltando el gran aporte de Verónica Guevara. A Hanzel Lacayo por contribuir en la elaboración y por su participación en parte de la investigación. A los profesores de la Facultad de Ciencia, Tecnología y Ambiente que con empeño apoyaron la profundización del conocimiento de estos temas.

\section{Referencias bibliográficas}

AISLABIE, J.; RICHARDS, K. \& BOUL, H. (1997). "Microbial degradation of DDT and its residues-a review". New Zealand Journal of Agricultural Research, Vol 40:269-282.

ÁLVAREZ, P.J.\& GUEVARA E. (2003).Biorremediación y atenuación natural de acuíferos contaminados por sustancias químicas peligrosas. Venezuela: Consejo de Desarrollo Científico y Humanístico, 1era Edición.

ATLAS, R. M. \& BARTHA, R. (2002). Ecología microbiana y microbiología ambiental. Madrid: Pearson Educación, S.A. 4ta edición.

ATSDR (AGENCY FOR TOXIC SUBSTANCES AND DISEASE REGISTRY). (2002) Toxicological profile for DDT, DDE, and DDD: Chemical and physical information. Disponible en: http://www.atsdr.cdc.gov/toxprofiles/tp35-c4.pdf. Consultado: 14 de octubre de 2007.

BARR, D. \& AUST, S. (1994). "Mechanisms white rot fungi use to degrade pollutants". Environmental science and technology. 28: 78A-87A.

BEULKE, S.; VAN BEINUM, W.; BROWN, C.; MITCHELL, M. \&WALKER A. (2005). "Evaluation of Simplifying Assumptions on Pesticide Degradation in Soil”. J Environ Qual 34:19331943.

BIODYNE (s.f.).Biotreatguidelines Disponible en: http://www.biodyne-srq.com. Consultado: 14 de febrero de 2007.

BIODYNE. (2008) Microbios Disponible en: http://www.biodyne-srq.com/pdtmic_esp.html . Consultado: 18 de enero de 2008.

BUMPUS, J. \& AUST, S. (1987). "Biodegradation of DDT [ 1,1,1-trichloro-2,2-bis(4chlorophenyl) ethane] by the white rot fungus Phanerochaete chrysosporium”. Applied and environmental microbiology. 53: 2001-2008.

CARRILLO, E.; RUIZ, A. \& YEOMANS, H. (2004). "Aislamiento, identificación y evaluación de un cultivo mixto de microorganismos con capacidad para degradar DDT". Revista Internacional de Contaminación Ambiental, abril-julio, año/vol. 20, número 002: 69-75.

CHACKE, C.; LOCKWOOD, J. \& ZABIK, M. (1966). “Chlorinated hydrocarbon pesticides: degradation by microbes". Science. 154(751): 893-5.

DIGITAL GLOBE (2008). Imágenes georeferenciadas. Google Earth (4.0.2737) [Software de cómputo]. Google. Obtenidas: 11 de enero de 2008.

EMBUSA (EMBAJADA DE LOS ESTADOS UNIDOS EN NICARAGUA) (1999). Nicaragua, diversification and growth (1945-1977).Managua:EMBUSA.

EPA (ENVIRONMENTAL PROTECTION AGENCY). (1984). EPA Method study 18 method 608-organochlorine pesticides and PCBs, EPA 600/4-84-061. Springfield: Vírginia National Technical Information Service. 
EUROPA TECHNOLOGIES LTD. (2008). Imágenes georeferenciadas. Google Earth (4.0.2737) [Software de cómputo]. Google. 11 de enero de 2008.

FRANCIS, J.; SPANGGORD, J.; OUCHI, I. \& BOHONOS, N. (1978). “Cometabolism of DDT Analogs by a Pseudomonas sp”. Appl Environ Microbiolo. February; 35(2): 354-367.

FOCHT, D. \& BRUNNER, W. (1985). "Kinetics of Biphenyl and Polychlorinated Biphenyl Metabolism in Soil”. Appl Environ Microbiol. 50(4): 1058-1063.

GALE, E. (1952). "The chemical activities of bacteria". Academic Press. Londres, Inglaterra.

GAGEL, B.; PIROTA, M.: PINKWA, M.; REINARTZ, P.; ZIMNY, M.; KAISER. H.; et al. (2007). "Po polography, contrast enhanced color duplex sonography (CDS), (18F) fluromisonidazole and $(18 \mathrm{~F})$ flurodeoxyglucose positron emission tomography: validated methods for the evaluation of therapy-relevant tumor oxygenation or only bricks in the puzzle of tumor hypoxia”. BMC Cancer. Jun 28; 7: 113.

HAY, A. \& FOCHT, D. (1998). "Cometabolism of 1,1-Dichloro-2,2-Bis(4-Chlorophenyl)Ethylene by Pseudomonas sp. acidovorans M3GY Grown on Biphenyl”. Appl. Environ. Microbiol. Vol. 64, 6: $2141-2146$.

HILL, R.; ASHLEY, D.; HAED, L.; NEEDHAM, L. \& PIRKLE, J. (1995). "P-Dichlorobenzene exposure among 1000 adults in the United States". Archives of Environmental Health. 50(4): 277-80.

KAMANAVALLI, C. \& NINNEKAR, H. (2004). "Biodegradation of DDT by a Pseudomonas sp". Current Microbiology. 48: 10-13

KALLMAN, B. \& ANDREWS, A. (1963). "Reductive Dechlorination of DDT to DDD by Yeast". Science. 141: 1050-1051.

MADIGAN, M.; MARTINKO, J.\&PARKER, J. (2004).Brock. Biología de los microorganismos. Madrid: Pearson Educación S.A., 10ma edición.

MEJÍAS, J. \& JEREZ , J. (2006) Guía para la toma de muestras de residuos de plaguicidas agua, sedimento y suelo. Disponible en: http:/www.sag.gob.cl/pls/portal/docs/PAGE/ PG_SAG_BIBLIOTECA/BIBL_MEDAMB/BIBLIO_MA GAMB/BIBLIO_MA_GAMB_DOCS/ GUIA\%20TOMA\%20MUESTRAS\%20PLAGUICIDAS.PDF. Consultado: $2 \overline{0}$ de enero de 2007.

PANTALEÓN (2007). Base de datos del Ingenio Monte Rosa. Chinandega, Nicaragua. [Intranet]. Consultado: marzo de 2007.

PATIL, K.; MATSUMURA, F., \& BOUSH, G. (1970). "Degradation of Endrin, Aldrin, and DDT by Soil Microorganisms”. Appl Microbiol. 19(5): 879-881.

PÉREZ, A. (2004). Aspectos conceptuales, análisis numéricos, monitoreo y publicación de datos sobre biodiversidad. Managua: Araucaria-MARENA. 1era edición.

SEUBERT, W. (1960). "Determination of isoprenoid compounds by microorganism I. Isolation and characterization of an isoprenoiddegrading bacterium Pseudomonas sp. citronellolis, new species". J Bacteriol. 79: 426-434.

SKOOG, D.; WEST, D.; HOLLER, F. \& CROUCH, S. (2001). Química analítica. México: McGraw Hill, 7 ma edición.

STANLAKE G. \& FINN R. (1982). "Isolation and charazterization of a phentachlorophenol degrading bacterium”. Appl. Environ. Microbiol. 44: 1421-1427.

TERRA METRICS (2008). Imágenes georeferenciadas. Google Earth (4.0.2737) [Software de cómputo]. Google. 11 de enero de 2008. 